\title{
Association of Fractional Flow on 3D-TOF-MRA with Cerebral Perfusion in Patients with MCA Stenosis
}

\author{
(D)X. Ge, (D) H. Zhao, (D) Z. Zhou, (D)X. Li, (D)B. Sun, (DH. Wu, (D). Wan, (D). Xu, (D).P. Villablanca, and (D) X. Liu
}

\begin{abstract}
BACKGROUND AND PURPOSE: Fractional flow measured on 3D-TOF-MRA was proposed to quantify cerebral hemodynamic changes in patients with artery stenosis. We investigated the association between fractional flow and cerebral perfusion changes in patients with symptomatic MCA stenosis.
\end{abstract}

MATERIALS AND METHODS: This prospective study was approved by the institutional review board, and all participants provided written informed consent. From June 2015 to May 2018, four hundred twenty-nine patients with symptomatic intracranial arterial stenosis were consecutively recruited and underwent conventional brain MR imaging, 3D-TOF-MRA, and brain CTP. A total of 91 patients with unilateral M1 segment stenosis of the MCA and a stenosis degree of 50\% 99\% were included in the analysis. Fractional flow was measured by comparing distal and proximal signal intensity changes across the stenosis on 3D-TOF-MRA. The cutoff value for fractional flow for discriminating between normal perfusion and hypoperfusion was obtained from the receiver operating characteristic curve. Associations between fractional flow and hypoperfusion were assessed using univariate and multivariate analyses.

RESULTS: The receiver operating characteristic curve showed a significant fractional flow threshold value at 0.90 (sensitivity, $70.1 \%$; $95 \%$ $\mathrm{Cl}, 55.9 \%-81.2 \%$; specificity, $69.6 \% ; 95 \% \mathrm{Cl}, 47.6 \%-84.1 \%)$. Participants with a fractional flow of $\leq 0.90$ were independently associated with cerebral hypoperfusion downstream from the stenosis site (adjusted OR, 3.68; 95\% Cl, 1.63-11.62; $P=.027$ ).

CONCLUSIONS: Fractional flow measured on 3D-TOF-MRA may serve as a noninvasive and practical tool for determining the cerebral hypoperfusion in patents with symptomatic MCA stenosis

ABBREVIATIONS: $\mathrm{FF}=$ fractional flow; ICAS $=$ intracranial arterial stenosis; $\mathrm{rMTT}=$ relative (affected side/contralateral side) $\mathrm{MTT}$; $\mathrm{rTTP}=$ relative (affected side/contralateral side) TTP; SAG-C = stressed autoregulation compensated; SAG-D = stressed autoregulation decompensated; $\mathrm{SI}=$ signal intensity

ntracranial arterial stenosis (ICAS) is one of the main causes of ischemic stroke or TIA. ${ }^{1}$ In addition to stenosis severity, several factors (such as plaque composition, collateral circulation status, and hemodynamic changes across the stenosis lesion) are closely associated with a risk of primary or recurrent stroke in patients with ICAS. ${ }^{2-4}$ Brain 3D-TOF-MRA has been widely used to diag-

Received December 11, 2018; accepted after revision May 3, 2019.

From the Departments of Radiology (X.G., H.Z., Z.Z., X. Li, B.S., J.X., X. Liu), Neurology (H.W.), and Neurosurgery (J.W.), Ren Ji Hospital, School of Medicine, Shanghai Jiao Tong University, Shanghai, P.R. China; and Department of Radiological Sciences and Neurosurgery (J.P.V.), David Geffen School of Medicine at University of California, Los Angeles, Los Angeles, California.

This research was supported by the National Natural Science Foundation of China (No. 81271575, 81401374, 81571630) and the Shanghai Jiao Tong University MedicalEngineering Cross-Cutting Research Project (No. YG2015MS53, YG2015QN36).

Please address correspondence to Xiaosheng Liu, MD, PhD, Department of Radiology, Ren Ji Hospital, School of Medicine, Shanghai Jiao Tong University, 160 Pujian Rd, Shanghai 200127, China; e-mail: miaxiaosheng@gmail.com

- Indicates open access to non-subscribers at www.ajnr.org

http://dx.doi.org/10.3174/ajnr.A6095 nose ICAS in clinical practice. Recently, fractional flow (FF) measured on 3D-TOF-MRA, a new imaging parameter, was proposed to quantify cerebral hemodynamic changes in patients with ICAS. $^{5-8}$

Although FF predicted the risk of stroke in patients with ICAS in a secondary analysis of the Stroke Outcomes and Neuroimaging of Intracranial Atherosclerosis (SONIA) trial, ${ }^{7}$ the underlying mechanism has not been clarified. Cerebral hemodynamic changes caused by ICAS presumably reduce the perfusion pressure in the corresponding downstream region. Because CTP is a fast and reliable imaging option to visualize cerebral perfusion, Cianfoni et $\mathrm{al}^{9}$ and Siemund et $\mathrm{al}^{10}$ used CTP to characterize dynamic perfusion changes in response to a progressive decline in perfusion pressure: When perfusion pressure drops into an ischemic range, cerebral perfusion is maintained through autoregulatory vasodilation, which manifests with a normal or slightly increased CBF, elevated CBV, and a corresponding increase in MTT. As perfusion pressure continues to decline, cerebral arterioles are unable to dilate further, and CBF and CBV decrease as a consequence, as MTT is further prolonged. TTP, 
an intuitive and straightforward parameter like MTT, is also extracted by some software packages.

Lan et $\mathrm{al}^{5}$ have explored the relationship between FF and cerebral perfusion in patients with ICAS and found that lower FF across the stenosis lesion could reflect delayed perfusion in the ipsilateral downstream region. However, their work was hindered by a long interval between MRA and CTP, a small sample size, and other potential confounders. In this study, we aimed to prospectively investigate the association between FF across the stenosis lesion and ipsilateral downstream perfusion on CTP in patients with symptomatic MCA stenosis and to assess the ability of the FF to discriminate cerebral hypoperfusion from normal perfusion in this clinical population.

\section{MATERIALS AND METHODS Participants}

The study was approved by the Ethic Committee of Shanghai Renji Hospital, and all participants provided written informed consent. From September 2015 to May 2018, we consecutively recruited patients with ischemic stroke or TIA who were diagnosed by a physician from the department of neurology of our hospital. Ischemic stroke was defined as an episode of neurologic dysfunction persisting $\geq 24$ hours or until death, caused by focal cerebral infarction on CT or MR imaging with other etiologies excluded. ${ }^{11}$ TIA was defined as a transient episode of neurologic dysfunction lasting $<24$ hours and caused by focal cerebral ischemia without a finding of acute infarction. The symptomatic MCA stenosis was defined as responsible for the neurologic dysfunction. All participants underwent conventional brain MR imaging, including 3D-TOF-MRA, and brain CTP within 1 day of enrollment. The location and degree of ICAS were evaluated on CTA from the arterial phases of CTP source data. Patients with unilateral MCA stenosis at the M1 segment and with a stenosis degree of $50 \% \sim 90 \%$ were included for analysis. Exclusion criteria were as follows: 1) cardioembolic stroke, 2) coexistent extracranial arterial stenosis with the stenosis degree of $>50 \%, 3$ ) tandem stenosis at the MCA by CTA and MRA, 4) MR signal intensity (SI) and absent CTA density loss at the M1 segment of the MCA due to complete occlusion, and 5) contraindications to MR imaging or CTP (heart pacemaker, metallic implant, severe claustrophobia, pregnancy, allergy, or an estimated glomerular filtration rate of $<30 \mathrm{~mL} / \mathrm{min} / 1.73 \mathrm{~m}^{2}$ ). Demographic and clinical characteristics of enrolled subjects were recorded. Anatomically severe and moderate stenosis was determined to be $70 \% \sim 99 \%$ and $50 \% \sim 69 \%$ luminal stenosis measured on CTA, respectively.

\section{Fractional Flow Measured on 3D-TOF-MRA}

All participants underwent conventional brain MR imaging and 3D-TOF-MRA using a 3T whole-body MR imaging scanner (Achieva; Philips Healthcare, Best, the Netherlands) with the following sequences-1) axial T1WI: $\mathrm{TR}=25 \mathrm{~ms}$, $\mathrm{TE}=2.3$ $\mathrm{ms} ; 2$ ) axial T2WI: TR $=3000 \mathrm{~ms}$, TE $=80 \mathrm{~ms} ; 3$ ) axial FLAIR: $\mathrm{TR}=7000 \mathrm{~ms}$, $\mathrm{TE}=120 \mathrm{~ms}$, TI $=2250 \mathrm{~ms}$; and 4 ) axial DWI: $\mathrm{TR}=2748 \mathrm{~ms}, \mathrm{TE}=97 \mathrm{~ms}$, b-value $=1000$. All axial MR images shared the following parameters: FOV $=230 \times 230 \mathrm{~mm}$, slice thickness $=5 \mathrm{~mm}$, acquisition matrix $=230 \times 230($ except DWI with the reconstructed matrix of $192 \times 192)$. The parameters of the 3D-TOF-MRA sequence were the following: slice thickness $=$ $1.2 \mathrm{~mm}, \mathrm{TR}=23 \mathrm{~ms}, \mathrm{TE}=3.5 \mathrm{~ms}$, flip angle $=18^{\circ}, \mathrm{FOV}=199 \times$ $199 \mathrm{~mm}$, acquisition matrix $=500 \times 332$. Eighteen MIPs with $10^{\circ}$ separation were constructed for anteroposterior and lateral views, respectively. The duration of MR imaging was 8 minutes 50 seconds.

FF was measured on an Extended MR Workspace 2.6.3.1 (Philips Healthcare) by 2 independent readers (X.G. and X. Liu with 3 and 15 years of experience in MR imaging interpretation, respectively), blinded to each other's readings and clinical data. According to the method proposed by Liebeskind et al, ${ }^{7}$ proximal and distal SIs across ICAS lesions were measured on the MIP image that showed the highest percentage of stenosis of the target lesion, using two 3-mm-length ROIs covering the entire vessel lumen (Fig 1). The boundary of the vessel edge was defined using a visual estimation of the full width at half maximum of the slicesensitivity profile. ${ }^{12}$ The background SI was estimated by the average SI in 2 circular ROIs (with an area of $15-20 \mathrm{~mm}^{2}$ ) placed symmetrically on both sides of the background on the same MIP image. The FF was calculated according to the following formula: $\mathrm{FF}=($ Distal SI - Background SI $) /($ Proximal SI - Background SI). To avoid the possible impact of window width and level settings on the lesion appearance, we standardized viewing thresholds between the 2 readers. Each lesion was measured twice by each reader; then, the average of the 2 measures was the FF value of the lesion from this reader.

Acute cerebral infarctions were identified as hyperintense lesions on the DWI and hypointense lesions on apparent diffusion coefficient maps. The volumes of the acute cerebral infarctions involving the MCA regions were measured by an independent radiologist (J.X. with 20 years of experience in MR imaging interpretation) twice for each patient with acute ischemic stroke on the Extended MR workstation. The volumes of acute cerebral infarctions were determined using the image of the affected slices with hyperintense areas visible from the $b=1000 \mathrm{~mm} / \mathrm{s}^{2}$ images. The sum of the volumes of the acute cerebral infarctions was recorded and averaged.

\section{Brain CT Perfusion}

All participants underwent cerebral CTP with a 64-detector row scanner (SOMATOM Sensation 64, Siemens, Forchheim, Germany). Scanning parameters of CTP were as follows: $100 \mathrm{kV}, 150$ $\mathrm{mAs}, 235 \times 199 \mathrm{~mm}$ collimation, 11.4-cm scan volume in the $\mathrm{z}$-axis, slice thickness $=5 \mathrm{~mm}$ using an adaptive spiral scanning technique ("shuttle mode"). Data were acquired at 40 time points separated by a repetition interval of 1.5 seconds. A total of $50 \mathrm{~mL}$ of nonionic iodinated contrast media, iohexol (Omnipaque 350; GE Healthcare, Piscataway, New Jersey), was injected at a rate of $5.0 \mathrm{~mL} / \mathrm{s}$, followed by a saline flush of $30 \mathrm{~mL}$ of sodium chloride at $5.0 \mathrm{~mL} / \mathrm{s}$ and a start delay of 5 seconds. The total CT dose index volume was $198.25 \mathrm{mGy}$, and the total dose-length product was $2258 \mathrm{mGy} \times \mathrm{cm}$. The CTP processing analysis was performed on a multimodality workstaion (Siemens syngo 2010B; Siemens) with the vendor-supplied Neuro-VPCT software based on the semiautomatic deconvolution algorithm "auto stroke." Motion correction, bone segmentation, estimation of arterial input, and venous output function were performed automatically before 

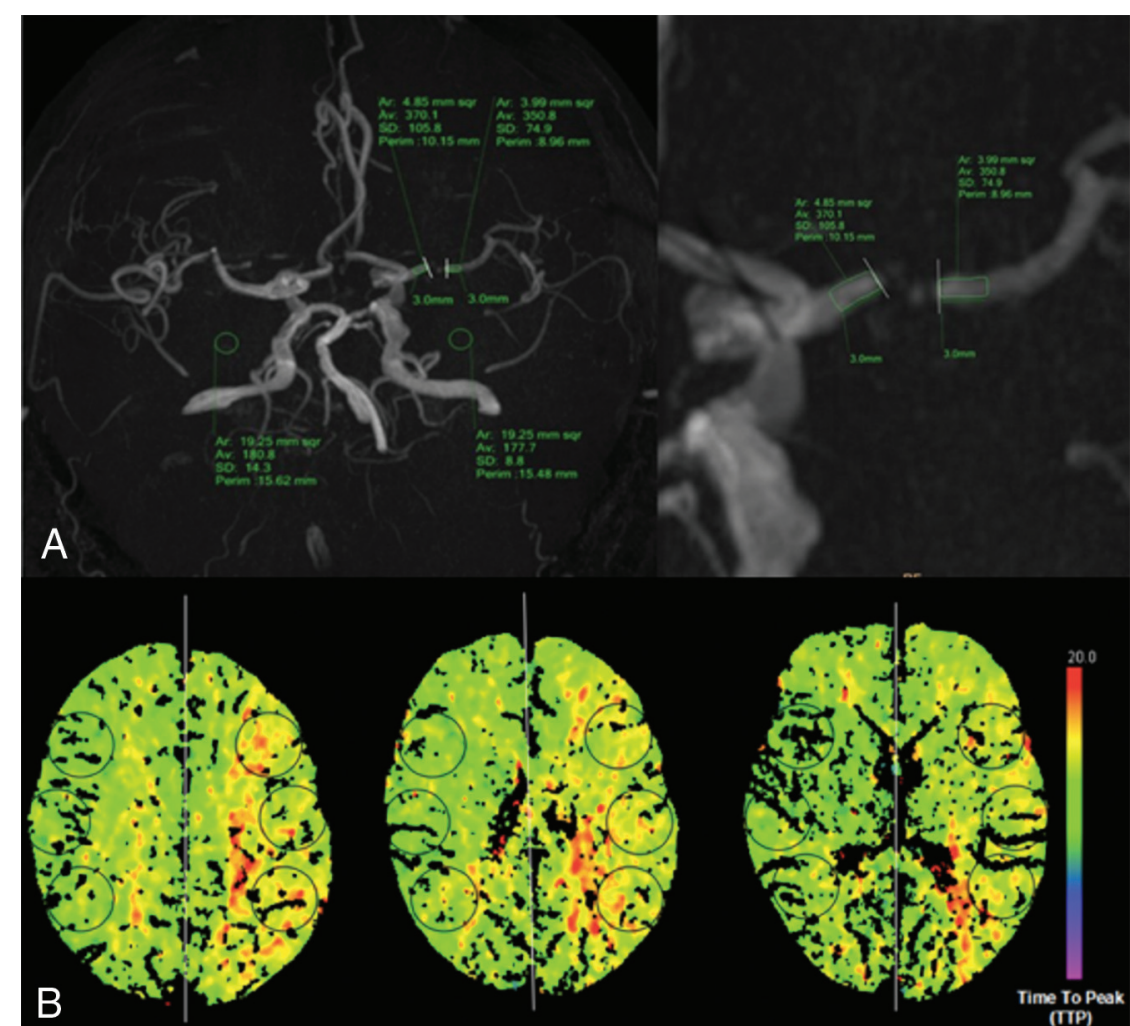

Centrum Semiovale Level

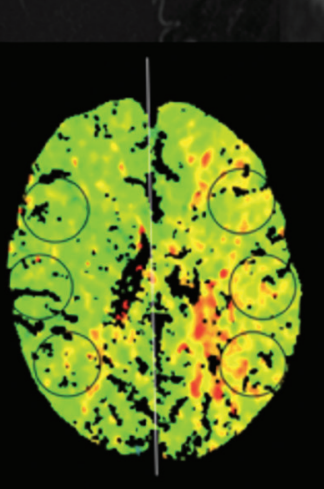

Corona Radiate Level

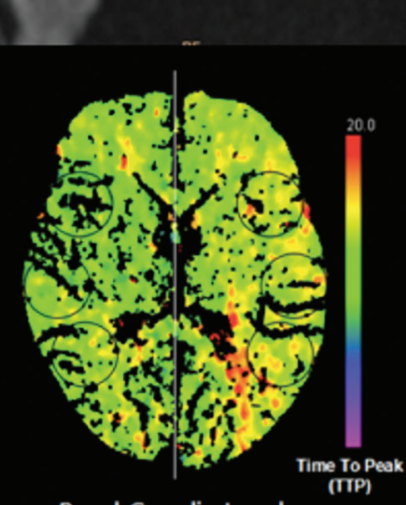

Basal Ganglia Level

FIG 1. Illustration of fractional flow measurement and cerebral perfusion assessment. $A$, FF measurement: measurement of signal intensities proximal and distal to a stenosis located at the left MCA on a 3D-MIP image of TOF-MRA. B, Cerebral perfusion assessment: 6 circular ROls were symmetrically drawn in the bilateral MCA territories at the centrum semiovale, corona radiata, and basal ganglia levels.

angiograms and color-coded perfusion parameter maps of MTT, TTP, CBF, and CBV were obtained.

The quantitative assessment of cerebral perfusion was performed by 2 independent radiologists (X. Li and Z.Z. with 4 and 10 years of experience in CTP interpretation, respectively) who were blinded to the FF outcome. Six circular ROIs $\left(8-12 \mathrm{~cm}^{2}\right)$ in either hemisphere were drawn manually and symmetrically in the bilateral MCA territories at 3 anatomic levels (the centrum semiovale, corona radiata, and basal ganglia levels) (Fig 1). The mean relative values of MTT (rMTT), TTP (rTTP), CBF, and CBV were calculated by the ratio of the mean value of the ROIs between the affected and contralateral sides. Ischemic penumbra was considered as a relative MTT increase of $>145 \%$ with increased TTP, mildly decreased CBF, and normal or even increased CBV. ${ }^{13}$ Core infarction was an affected CBF reduction of $>31 \%$ of the contralateral side, with increased MTT and TTP and decreased CBV. ${ }^{14}$ Radiologists needed to refer to the DWI to avoid missing small lacunar infarcts on color-coded perfusion parameter maps. Hypoperfusion was defined as ischemic penumbra and/or core infarction in the downstream region of the lesion and was graded as 1 of 2 stages: the stressed autoregulation compensated (SAG-C) stage (ischemic penumbra without core infarction) and the stressed autoregulation decompensated (SAG-D) stage (with core infarction) (Fig 2).

The degree of luminal stenosis and stenosis lengths were blindly measured twice by the same reader (B.S. with 5 years of experience in brain CT interpretation) for each patient, and the

values were averaged. The degree of stenosis on CTA was calculated by following equation: Percentage Stenosis = $\left[1-\left(D_{\text {stenosis }} / D_{\text {normal }}\right)\right] \times 100 \%$. $\mathrm{D}_{\text {stenosis }}$ is the diameter of vessel at the side of stenosis. $\mathrm{D}_{\text {normal }}$ is the diameter of the vessel distal to the stenosis. ${ }^{15}$ Anatomically moderate and severe stenoses were determined as 50\%-69\% luminal stenosis and 70\%-99\% luminal stenosis, respectively.

Collateral circulation in MCA territories was assessed on the basis of a 6-point ordinal scale proposed by Menon et al. ${ }^{16}$ The 15 th (the peak arterial phase), 20th, and 25th phases of the CTP original images were chosen to grade collateral scores. Two independent radiologists (H.Z. and B.S. with 10 and 5 years of experience in brain CT interpretation, respectively) who were blinded to the data of adjusted FF and CT perfusion compared the pial arterial filling between symptomatic and contralateral hemispheres in 3 CTP phases and then performed collateral scoring.

\section{Statistical Analysis}

Data were presented as mean \pm SD and median or count (percentage) as appropriate. The 1-way random intraclass correlation coefficient was performed to assess the interreader reproducibility of the FF. Mean FFs of 2 readers were compared using paired $t$ tests. Interreader agreement on collateral scoring and hypoperfusion decision-making were assessed by the Cohen $\kappa$ test. The $\kappa$ value or intraclass correlation coefficient was interpreted as fair $(0.20-0.40)$, moderate $(0.41-$ $0.60)$, good (0.61-0.80), or excellent (0.81-1.00) agreement. Receiver operating characteristic curves were used to calculate the sensitivity and specificity of the FF for distinguishing normal perfusion and hypoperfusion. The threshold was set according to the Youden index. The association between the FF and hypoperfusion was assessed using the logistic regression model. First, univariable association was determined, and a multivariable model was then used to adjust potential confounders with $P$ values of $<.05$ in the univariable analysis. Correlations between FF and perfusion parameters, degree of luminal stenosis, stenosis lengths, the volumes of acute cerebral infarctions, and the degree of hypoperfusion were evaluated by either the Pearson or Spearman correlation coefficient. The 1-way ANOVA test followed by the least significant difference test was applied for multiple comparisons of FF among different degrees of cerebral hypoperfusion. Differences of FF between normal perfusion and hypoperfusion groups were analyzed by the independent-samples $t$ test. SPSS software (Version 19.0; IBM, Armonk, New York) was used for statistical analysis. A $P$ value $<.05$ was considered statistically significant. 


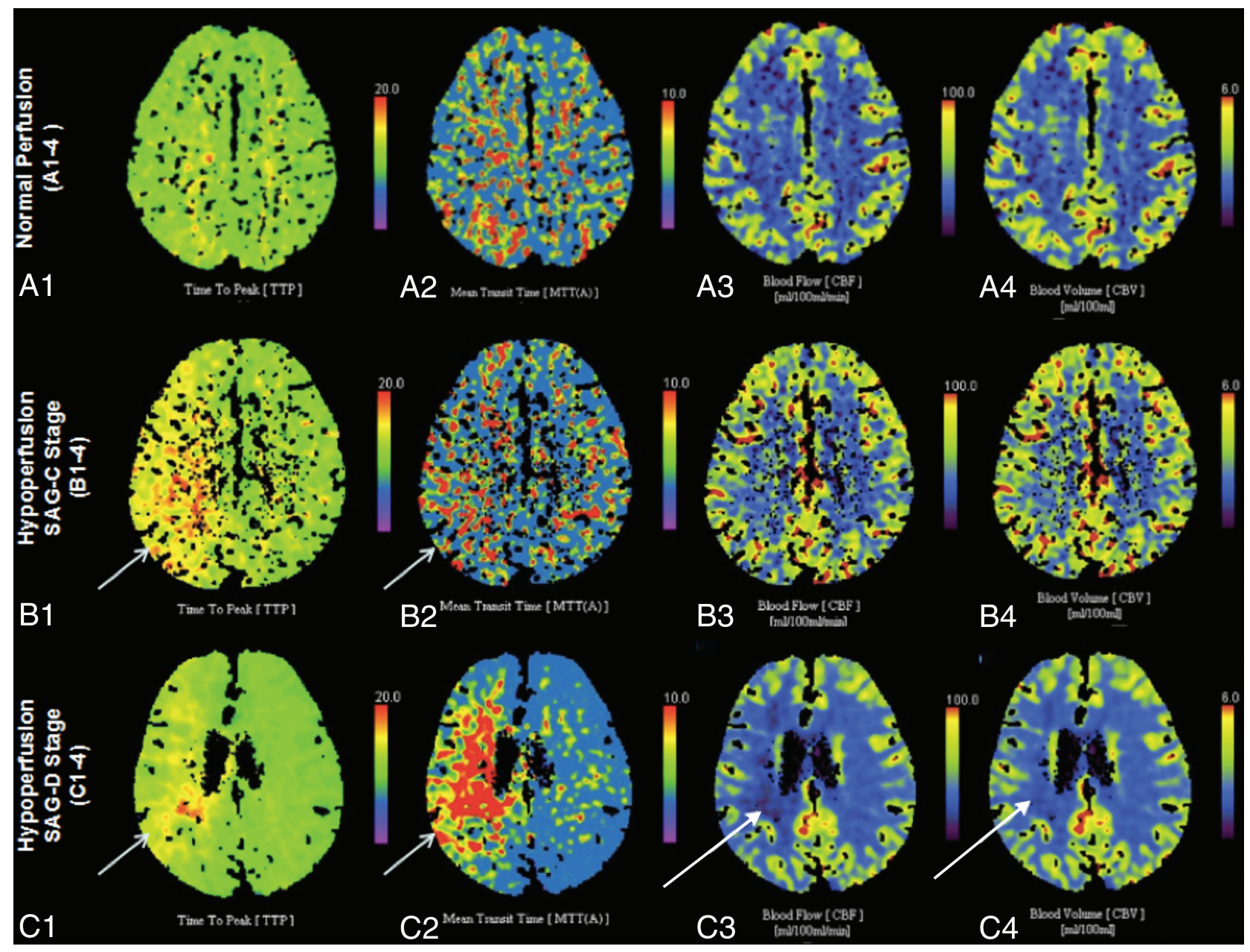

FIG 2. Color-coded perfusion parameter maps show the degree of cerebral hypoperfusion. A1-4, Normal perfusion. B1-4, Hypoperfuison on the stressed autoregulation compensated stage (ischemic penumbra without core infarction). Cl-4, Hypoperfuison on the SAG-D stage (ischemic penumbra with core infarction). Short arrows show ischemic penumbra regions (increased TTP and MTT, normal CBF and CBV). The long arrows show core infarction regions (increased TTP and MTT, decreased CBF and CBV).

\section{RESULTS}

\section{Characteristics of Participants}

In total, 91 eligible participants (28 women and 63 men; mean age, $59.0 \pm 9.6$ years) were included in the analysis. Patients with stroke spanned the acute-to-chronic stage (median time since stroke, 22.7 days; interquartile range, $12.3 \sim 36.1$ days). The percentage of patients with MCA stenosis was $89.0 \%(n=81)$ on the symptomatic side and $11.0 \%(n=10)$ on the asymptomatic side. A total of 338 participants were excluded due to cardioembolic stroke $(n=21)$, intracranial artery occlusion $(n=68)$, ICAS involving the posterior circulation or intracranial segment of the internal carotid artery $(n=89)$, multiple ICAS or extracranial arterial stenosis $(n=118)$, tandem stenosis at the MCA $(n=37)$, or poor image quality due to motion artifacts $(n=5)$. Participants with hypoperfusion were significantly different from those with normal perfusion in current smoking, anatomic severity of the lesion, collateral scoring, and $\mathrm{FF}(P=.001, P=.004, P=.002$, and $P=.000$, respectively) (Table 1 ).

Mean FFs were not significantly different between 2 readers $(P=.309$, mean difference $=.008)$. The interreader agreement was good for FF (intraclass correlation coefficient $=0.92$ ), hypo- perfusion assessment $(\kappa=0.93)$, and collateral circulation scoring $(\kappa=0.90)$.

\section{FF Cutoff Value Determined from the Receiver Operating Characteristic Curve}

Twenty-nine (31.9\%) participants had normal perfusion, and 62 (68.1\%) had hypoperfusion involving the ipsilateral MCA region. The mean FF value of the hypoperfusion group was significantly lower than that of normal-perfusion group $(0.7 \pm 0.2$ versus $0.9 \pm 0.2 ; P=.000)$. The receiver operating characteristic curve of FF to distinguish normal perfusion and hypoperfusion is shown in Fig 3. The area under the curve was 0.702 . For the FF, the Youden index was greatest when a threshold value of 0.90 was applied (sensitivity, 70.1\%; 95\% CI, 55.9\%-81.2\%; specificity, 69.6\%; 95\% CI, 47.6\%-84.1\%).

\section{Association between FF and Cerebral Perfusion}

Table 2 shows the results of the logistic regression analyses of FF. In univariate analysis, there were significant differences between normal perfusion and hypoperfusion in the FF, collateral score, and current smoking $(P=.046, P=.049$, and $P=.043$, respec- 
Table 1: Characteristics of patients and lesions

\begin{tabular}{|c|c|c|c|}
\hline & $\begin{array}{c}\text { With Normal } \\
\text { Perfusion on } \\
\text { Brain CTP }(n=29)\end{array}$ & $\begin{array}{c}\text { With Hypoperfusion } \\
\text { on Brain } \\
\text { CTP }(n=62)\end{array}$ & $\begin{array}{c}P \\
\text { Value }\end{array}$ \\
\hline Age (mean) (yr) & $58.8 \pm 9.2$ & $61.5 \pm 10.9$ & .237 \\
\hline Male sex (No.) (\%) & $16(17.6 \%)$ & $47(51.6 \%)$ & .261 \\
\hline Current smoker (No.) (\%) & $4(4.4 \%)$ & $30(33.0 \%)$ & .001 \\
\hline Hypertension history (No.) (\%) & $13(14.3 \%)$ & $38(41.8 \%)$ & .073 \\
\hline Diabetes mellitus history (No.) (\%) & $5(5.5 \%)$ & $19(20.9 \%)$ & .204 \\
\hline \multicolumn{4}{|l|}{ Blood pressure (mean) $(\mathrm{mm} \mathrm{Hg})$} \\
\hline $\mathrm{SBP}$ & $139.6 \pm 25.9$ & $140.7 \pm 24.1$ & .874 \\
\hline DBP & $79.3 \pm 14.3$ & $83.1 \pm 13.9$ & .283 \\
\hline Fasting blood glucose (mean) (mmol/L) & $5.5 \pm 1.1$ & $6.4 \pm 2.5$ & .069 \\
\hline \multicolumn{4}{|l|}{ Cholesterol (mean) (mmol/L) } \\
\hline Total cholesterol & $4.1 \pm 0.8$ & $4.0 \pm 1.2$ & .814 \\
\hline $\mathrm{HDL}$ & $1.1 \pm 0.3$ & $1.1 \pm 0.4$ & .988 \\
\hline LDL & $2.2 \pm 0.7$ & $2.2 \pm 1.0$ & .954 \\
\hline Triglycerides & $1.7 \pm 0.9$ & $1.5 \pm 0.9$ & .494 \\
\hline \multicolumn{4}{|l|}{ Admitting diagnosis (No.) (\%) } \\
\hline Transient ischemic attack & 10 (11.0\%) & $11(12.1 \%)$ & .916 \\
\hline Acute ischemic stroke & $8(8.8 \%)$ & $26(28.6 \%)$ & .086 \\
\hline Chronic ischemic stroke & $11(12.1 \%)$ & $25(27.5 \%)$ & .541 \\
\hline Time from onset to CT/MRI (mean) (day) & $29.1 \pm 19.6$ & $24.0 \pm 18.9$ & .408 \\
\hline Median (interquartile range $25 \%-75 \%$ ) & $24(15.5 \sim 46)$ & $22(11 \sim 30)$ & .536 \\
\hline \multicolumn{4}{|l|}{ Anatomic severity of lesion on CTA (No.) (\%) } \\
\hline Severe & $10(11.0 \%)$ & $49(53.8 \%)$ & .004 \\
\hline Moderate & $19(20.9 \%)$ & $13(14.3 \%)$ & .674 \\
\hline Stenosis length (mean) (mm) & $4.7 \pm 2.5$ & $4.9 \pm 2.7$ & .795 \\
\hline Collateral scoring on CTA & $4.5 \pm 0.4$ & $3.7 \pm 1.5$ & .002 \\
\hline Fractional flow on MRA & $0.9 \pm 0.2$ & $0.7 \pm 0.2$ & .000 \\
\hline
\end{tabular}

Note:- - HDL indicates high density lipoprotein; LDL, low density lipoprotein; SBP, systolic blood pressure; DBP, diastolic blood pressure.

\section{ROC Curve}

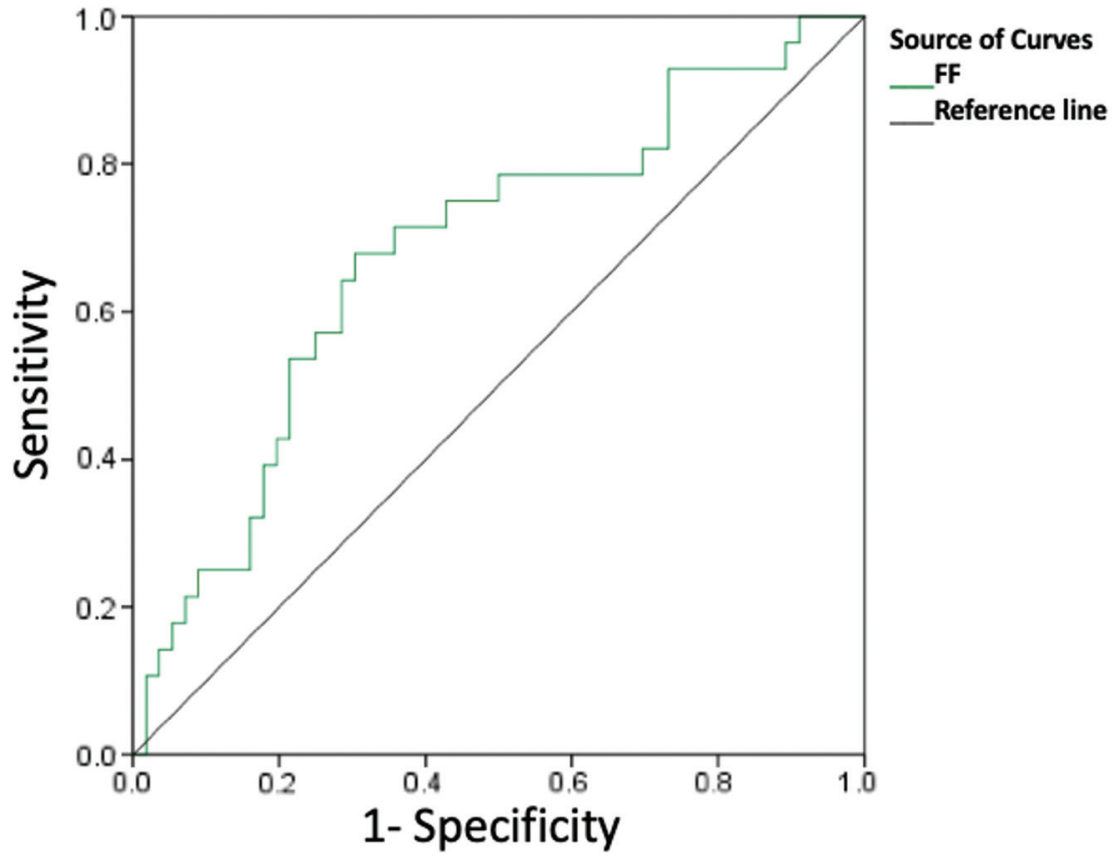

FIG 3. Receiver operating characteristic (ROC) curve of fractional flow to differentiate normal perfusion and hypoperfusion.

tively). In multivariate analysis, an FF of $\leq 0.90$ was independently associated with cerebral hypoperfusion in the region downstream from a stenosis site (adjusted OR, 3.68; 95\% CI, 1.63-11.62; $P=$ .027 ) and after correcting for collateral score and current smoking ICAS.
(OR, 0.26; 95\% CI, 0.07-0.97; $P=$ .041 ; OR, 7.20; 95\% CI, 1.43-36.40; $P=.017)$.

FF was different among normal (29, $31.9 \%)$, SAG-C $(22,24.2 \%)$, and SAG-D $(40,43.9 \%)$ perfusion stages $(P=.021)$, and a negative correlation was found between FF and different cerebral perfusion stages $(\rho=-0.325, P=.010)$. The mean FF at the normal stage $(0.93 \pm$ 0.15) was higher than that at SAG-C stage $(0.86 \pm 0.21, P=.024)$ or at the SAG-D stage $(0.72 \pm 0.18, P=.011)$. The mean FFs at SAG-C and SAG-D stages were not different $(P=.534)$ (Fig 4). In terms of the perfusion parameters, the FF was correlated with $\operatorname{rTTP}(r=$ $-0.52, P<.001)$, rMTT $(r=-0.57$, $P<.001)$, and relative $\mathrm{CBF}(r=0.21$, $P=.043)$, but not with relative $\mathrm{CBV}$ $(r=0.16, P=.143)$ in all eligible participants (Table 3 ). Stratification by the collateral score showed that the correlations between FF and rTTP and rMTT in poor collateral circulation (collateral score 0-3 group; $r=$ $-0.485, P=.001 ; r=-0.414, P=$ $.001)$ were stronger than those in good collateral circulation (collateral score $4-5$ group; $r=-0.250, P=.004 ; r=$ $-0.361, P=.002)$.

Neither the degree of luminal stenosis nor stenosis lengths were significantly correlated with FF $(r=-0.217$, $P=.089 ; r=-0.163, P=.315)$. In patients with acute cerebral infarctions, there was no significant correlation between FF and the volume of acute cerebral infarctions $(r=-0.119, P=$ .076).

\section{DISCUSSION}

In this study, there was an independent association between the FF across the stenosis site and cerebral hypoperfusion in the downstream region in participants with ICAS at the M1 segment of the MCA. FF could discriminate cerebral hypoperfusion from normal perfusion at a cutoff value of 0.90 with relatively high sensitivity and specificity. The interreader reproducibility of the FF measurement was good, a finding consistent with the results of a prior study. ${ }^{17}$ These findings suggest that FF measured on 3D-TOF-MRA could be adopted as a noninvasive and practical tool to determine the hemodynamic change in patients with 
Table 2: Association of participant characteristics with cerebral hypoperfusion in univariable and multivariable logistic regression models

\begin{tabular}{|c|c|c|c|c|}
\hline & \multicolumn{2}{|c|}{ Univariable Model } & \multicolumn{2}{|c|}{ Multivariable Model } \\
\hline & $P$ Value & OR $(95 \% \mathrm{Cl})$ & $P$ Value & OR $(95 \% \mathrm{Cl})$ \\
\hline Age (per 1-yr increase) & .542 & $0.98(0.90-1.05)$ & & \\
\hline Male vs female & .254 & $0.38(0.07-2.01)$ & & \\
\hline Current smoker (Y vs N) & .043 & $8.06(1.06-60.71)$ & .017 & $7.20(1.43-36.40)$ \\
\hline Hypertension history (Y vs N) & .072 & $3.99(0.89-18.06)$ & & \\
\hline Diabetes mellitus history (Y vs N) & .847 & $1.18(0.21-6.51)$ & & \\
\hline SBP (per 1-mm Hg increase) & .141 & $0.96(0.91-1.01)$ & & \\
\hline DBP (per l-mm Hg increase) & .615 & $1.02(0.94-1.16)$ & & \\
\hline Fasting blood glucose (per 1-mmol/L increase) & .081 & $1.75(0.93-3.14)$ & & \\
\hline HDL (per 1-mmol/L increase) & .427 & $3.19(0.14-75.54)$ & & \\
\hline LDL (per 1-mmol/L increase) & .299 & $3.37(0.19-60.23)$ & & \\
\hline Triglycerides (per 1-mmol/L increase) & .180 & $0.53(0.23-1.41)$ & & \\
\hline Total cholesterol (per 1-mmol/L increase) & .300 & $0.17(0.07-4.72)$ & & \\
\hline Time from onset to CT/MRI (per 1-day lapse) & .451 & $0.98(0.95-1.03)$ & & \\
\hline Anatomic severity of ICAS on CTA (severe vs moderate) & .274 & $0.59(0.48-12.86)$ & & \\
\hline Stenosis length (per 1-mm increase) & .262 & $0.54(0.45-13.06)$ & & \\
\hline Collateral score on CTA (per 1-score increase) & .049 & $0.28(0.06-1.22)$ & .041 & $0.26(0.07-0.97)$ \\
\hline Fractional flow on MRA ( $\leq 0.90$ vs $>0.90)$ & .046 & $7.28(1.03-51.35)$ & .027 & $3.68(1.63-11.62)$ \\
\hline
\end{tabular}

Note:-Y indicates yes; N, no; HDL, high density lipoprotein; LDL, low density lipoprotein; SBP, systolic blood pressure; DBP, diastolic blood pressure.

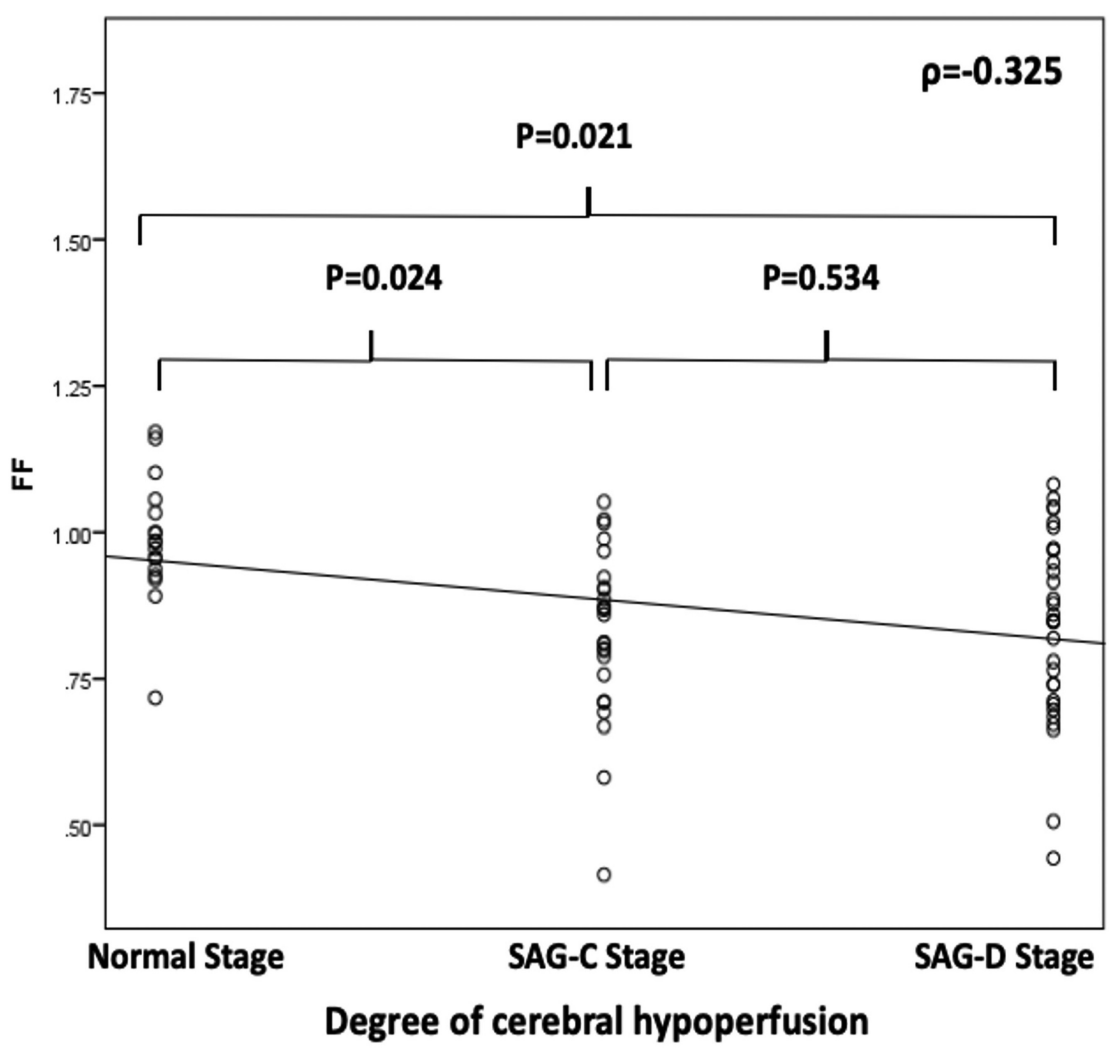

FIG 4. Correlations between fractional flow and the degree of cerebral hypoperfusion. hypoperfusion from normal perfusion at the cutoff value of 0.90 . In addition, we used reasonable ROIs in the bilateral MCA territories on the CTP maps, examined a larger sample size, and took more confounders into consideration, including stenosis severity, collateral circulation scores, and the interval time between CT and MR imaging.

Liebeskind et $\mathrm{al}^{7}$ reported that $\mathrm{FF}$ could predict the risk of stroke in patients with ICAS. The underlying mechanism might be the mediation of cerebral perfusion impairment caused by a hemodynamic change. ${ }^{18,19}$ Most interesting, our FF cutoff value (0.90) in the discrimination of hypoperfusion from normal perfusion is almost the same as that (0.9) in the prediction of stroke risk in patients with MCA stenosis. ${ }^{7}$ A further prospective cohort study is needed to confirm the underlying mechanism by taking stroke events as the outcome and analyzing both FF and cerebral perfusion at baseline. According to the flow-related enhancement theory, an SI change of vessel lumen across the stenosis site on 3D-TOF-MRA is nonlinearly
Previous studies have reported that 3D-TOF-MRA was not only able to diagnose ICAS but also provide information on hemodynamic changes due to stenotic lesions. Leng et $\mathrm{al}^{6}$ reported that SI changes across a stenosis on 3D-TOF-MRA were correlated with acute infarct volume on DWI. Lan et $\mathrm{al}^{5}$ found that reduced SI was associated with delayed ipsilateral cerebral perfusion. However, the threshold FF value for determining hemodynamically significant ICAS is still unknown. In this study, the dichotomous FF was used in the analysis of its association with cerebral perfusion. We found that FF could discriminate cerebral correlated with the change of blood flow velocity caused by luminal stenosis. ${ }^{20-22}$ A nonlinear formula for FF calculation still awaits development.

We found that FF was significantly correlated with some of the perfusion parameters that are sensitive to perfusion pressure. Our results on the correlation between FF and rMTT were also confirmed in a prior study. ${ }^{5}$ The possible mechanisms may be that TTP and MTT were sensitive to perfusion pressure, and CBV and CBF were mainly influenced by the autoregulation mechanism and collateral circulation. ${ }^{9}$ In addition, the flow direction postste- 
Table 3: Correlations between FF and perfusion parameters in different subgroups

\begin{tabular}{|c|c|c|c|c|c|c|c|c|c|c|}
\hline \multirow[b]{3}{*}{ Groups } & \multicolumn{4}{|c|}{ Collateral Score } & \multicolumn{4}{|c|}{ Current Smoking } & \multicolumn{2}{|c|}{ All } \\
\hline & \multicolumn{2}{|c|}{$\begin{array}{c}0 \sim 3 \\
(n=26 ; 28.6 \%)\end{array}$} & \multicolumn{2}{|c|}{$\begin{array}{c}4 \sim 5 \\
(n=65 ; 71.4 \%)\end{array}$} & \multicolumn{2}{|c|}{$\begin{array}{c}\text { Yes } \\
(n=34 ; 37.4 \%)\end{array}$} & \multicolumn{2}{|c|}{$\begin{array}{c}\text { No } \\
(n=57 ; 62.6 \%)\end{array}$} & \multicolumn{2}{|c|}{$(n=91 ; 100 \%)$} \\
\hline & $r$ & $P$ & $r$ & $P$ & $r$ & $P$ & $r$ & $P$ & $r$ & $P$ \\
\hline rTTP & -0.485 & .001 & -0.250 & .004 & -0.509 & .018 & -0.502 & .017 & -0.517 & .000 \\
\hline rMTT & -0.414 & .001 & -0.361 & .002 & -0.454 & .019 & -0.507 & .011 & -0.569 & .000 \\
\hline $\mathrm{rCBF}$ & -0.103 & .739 & 0.079 & .592 & 0.270 & .046 & 0.203 & .167 & 0.213 & .043 \\
\hline rCBV & 0.033 & .914 & 0.004 & .977 & -0.172 & .400 & 0.128 & .384 & 0.155 & .143 \\
\hline
\end{tabular}

Note:- $r C B V$ indicates relative $C B V ;$ rCBF, relative $C B F$.

nosis may be reversed and may create a low signal on 3D-TOFMRA distal to the stenosis in case of good collateral flow, providing a false impression that the stenosis is hemodynamically relevant. The FF had a limited ability to detect the preservation or a slight decrease in relative $\mathrm{CBF}$ and relative $\mathrm{CBV}$. Thus, there was no significant difference in FF between SAG-C and SAG-D stages in our study, SAG-C and SAG-D stages graded according to the decrease of $\mathrm{CBF}$ and $\mathrm{CBV}$.

Collateral blood is one of the key factors in maintaining normal cerebral perfusion in ischemic regions when primary sources of flow have become compromised. ${ }^{23}$ To reduce the effect of cerebral collateral circulation on the results, we chose the unilateral MCA stenosis at the M1 segment, which could avoid the compensation of communicating arteries of the circle of Willis. In addition, we also took the secondary collateral pathways into account, consisting of intracranial leptomeningeal anastomoses. Also, we found that correlations between FF and rTTP and rMTT in the collateral score $0-3$ group were stronger than those in the collateral score 4-5 group. The reason may be that hemodynamic changes across the ICAS lesion may directly influence downstream perfusion when collateral blood is poor.

Strengths of this study included the prospective design, relatively large sample size, short interval between MR imaging and CT, and adjusting clinical or imaging confounders related to the cerebral perfusion. This study also had several limitations. First, we recruited only participants with ICAS at the unilateral M1 segments of the MCA. Therefore, generalization of the conclusions from the current study may be limited by the selection bias. The application of FF in patients with ICAS at multiple sites will be further explored in future studies. Second, our sample size was not large enough for subgroup analysis, so a prospective study with more participants is needed. Furthermore, several factors could affect the SI across the stenosis site on TOF-MR imaging, such as vessel orientation, vessel tortuosity, natural vessel-caliber change, and mineralized atherosclerotic plaques, which could induce potential bias. We did not evaluate the possible impact of variable imaging and postprocessing parameters, such as slice thickness, flip angle, and reconstruction interval on FF values, and we emphasize that the cutoff values we obtained may be specific to the imaging protocol used in this study. Finally, although CTP is a widely available technique in the clinical application, the accuracy of CTP results is challenging and is affected by multiple factors such as arterial input function selection, arterial curves, and less sensitivity for lacunar infarcts.

\section{CONCLUSIONS}

This study demonstrates that an FF of $<0.90$ measured on 3DTOF-MRA can independently predict the risk of cerebral hypo- perfusion in the region downstream from the MCA stenosis. Its ability to discriminate between normal perfusion and hypoperfusion in patients with ischemic stroke or TIA suggests that it may serve as a simple tool for risk stratification in routine clinical practice.

Disclosures: Xiaoqian Ge-RELATED: Grant: National Natural Science Foundation of China (No. 81271575, 81401374, 81571630) and the SJTU Medical-Engineering CrossCutting Research Project (No. YG2015MS53, YG2015QN36). * Huilin Zhao-RELATED: Grant: National Natural Science Foundation of China (grants 81571630, 81401374), Shanghai Jiao Tong University (YG2016MS56), and Renji Hospital (RJZZ18-001).* Zien Zhou-UNRELATED: Grants/Grants Pending: University of New South Wales Sydney, Comments: Scientia PhD Scholarship (2018-2022). Xiao Li-RELATED: Grant: National Natural Science Foundation of China (No. 81271575, 81401374, 81571630) and the SJTU Medical-Engineering Cross-Cutting Research Project (No. YG2015MS53, YG2015QN36).* Beibei Sun—RELATED: Grant: This research was supported by the National Natural Science Foundation of China (No. 81271575, 81401374, 81571630) and the SJTU Medical-Engineering Cross-Cutting Research Project (No. YG2015MS53, YG2015QN36).* Hengqu Wu-RELATED: Grant: National Natural Science Foundation of China (No. 81271575, 81401374, 81571630) and the SJTU Medical-Engineering Cross-Cutting Research Project (No. YG2015MS53, YG2015QN36). * Xiaosheng LiuRELATED: Grant: the National Natural Science Foundation of China (No. 81271575, 81401374, 81571630) and the SJTU Medical-Engineering Cross-Cutting Research Project (No. YG2015MS53, YG2015QN36).* *Money paid to the institution.

\section{REFERENCES}

1. Holmstedt CA, Turan TN, Chimowitz MI. Atherosclerotic intracranial arterial stenosis: risk factors, diagnosis, and treatment. Lancet Neurol 2013;12:1106-14 CrossRef Medline

2. Pu Y, Lan L, Leng X, et al. Intracranial atherosclerosis: from anatomy to pathophysiology. Int J Stroke 2017;12:236-45 CrossRef Medline

3. Lan L, Leng X, Ip V, et al. Prolonged perfusion predicts recurrent ischemic stroke but not transient ischemic attack in patients with symptomatic intracranial stenosis. Curr Neurovasc Res 2017;14: 149-57 CrossRef Medline

4. Lu SS, Ge S, Su CQ, et al. MRI of plaque characteristics and relationship with downstream perfusion and cerebral infarction in patients with symptomatic middle cerebral artery stenosis. J Magn Reson Imaging 2018;48:66-73 CrossRef Medline

5. Lan L, Leng X, Abrigo J, et al. Diminished signal intensities distal to intracranial arterial stenosis on time-of-flight MR angiography might indicate delayed cerebral perfusion. Cerebrovasc Dis 2016;42: 232-39 CrossRef Medline

6. Leng X, Wong KS, Soo Y, et al. Magnetic resonance angiography signal intensity as a marker of hemodynamic impairment in intracranial arterial stenosis. PLoS One 2013;8:e80124 CrossRef Medline

7. Liebeskind DS, Kosinski AS, Lynn MJ, et al. Noninvasive fractional flow on MRA predicts stroke risk of intracranial stenosis. J Neuroimaging 2015;25:87-91 CrossRef Medline

8. Liebeskind DS, Feldmann E. Fractional flow in cerebrovascular disorders. Intervent Neurol 2012;1:87-99 CrossRef

9. Cianfoni A, Colosimo C, Basile M, et al. Brain perfusion CT: principles, technique and clinical applications. Radiol Med 2007;112: 1225-43 CrossRef Medline 
10. Siemund R, Cronqvist M, Andsberg G, et al. Cerebral perfusion imaging in hemodynamic stroke: be aware of the pattern. Interv Neuroradiol 2009;15:385-94 CrossRef Medline

11. Sacco RL, Kasner SE, Broderick JP, et al; American Heart Association Stroke Council, Council on Cardiovascular Surgery and Anesthesia, Council on Cardiovascular Radiology and Intervention, Council on Cardiovascular and Stroke Nursing, Council on Epidemiology and Prevention, Council on Peripheral Vascular Disease, Council on $\mathrm{Nu}-$ trition, Physical Activity and Metabolism. An updated definition of stroke for the 21st century: a statement for healthcare professionals from the American Heart Association/American Stroke Association. Stroke 2013;44:2064-89 CrossRef Medline

12. Greene TC, Rong XJ. Evaluation of techniques for slice sensitivity profile measurement and analysis. J Appl Clin Med Phys 2014;15: 4042 CrossRef Medline

13. Wintermark M, Flanders AE, Velthuis B, et al. Perfusion-CT assessment of infarct core and penumbra: receiver operating characteristic curve analysis in $\mathbf{1 3 0}$ patients suspected of acute hemispheric stroke. Stroke 2006;37:979-85 CrossRef Medline

14. Campbell BC, Christensen S, Levi CR, et al. Cerebral blood flow is the optimal CT perfusion parameter for assessing infarct core. Stroke 2011;42:3435-40 CrossRef Medline

15. Samuels OB, Joseph GJ, Lynn MJ, et al. A standardized method for measuring intracranial arterial stenosis. AJNR Am J Neuroradiol 2000;21:643-46 Medline

16. Menon BK, D’Esterre CD, Qazi EM, et al. Multiphase CT angiography: a new tool for the imaging triage of patients with acute ischemic stroke. Radiology 2015;275:510-20 CrossRef Medline

17. Leng X, Ip HL, Soo Y, et al. Interobserver reproducibility of signal intensity ratio on magnetic resonance angiography for hemodynamic impact of intracranial atherosclerosis. J Stroke Cerebrovasc Dis 2013;22:e615-19 CrossRef Medline

18. Markus H, Cullinane M. Severely impaired cerebrovascular reactivity predicts stroke and TIA risk in patients with carotid artery stenosis and occlusion. Brain 2001;124:457-67 CrossRef Medline

19. Jongen LM, Hendrikse J, Moll FL, et al. Cerebral perfusion affects the risk of ischemia during carotid artery stenting. Cerebrovasc Dis 2010;29:538-45 CrossRef Medline

20. Kodama T, Watanabe K. Influence of imaging parameters, flow velocity, and pulsatile flow on three-dimensional time-of-flight MR angiography: experimental studies. Eur J Radiol 1997;26:83-91 CrossRef Medline

21. Wilcock DJ, Jaspan T, Worthington BS. Problems and pitfalls of 3-D TOF magnetic resonance angiography of the intracranial circulation. Clin Radiol 1995;50:526-32 CrossRef Medline

22. Mustert BR, Williams DM, Prince MR. In vitro model of arterial stenosis: correlation of MR signal dephasing and trans-stenotic pressure gradients. Magn Reson Imaging 1998;16:301-10 CrossRef Medline

23. Ginsberg MD. The cerebral collateral circulation: Relevance to pathophysiology and treatment of stroke. Neuropharmacology 2018; 134:280-92 CrossRef Medline 